\title{
Killing kinetics of fidaxomicin and its major metabolite, OP-1118, against Clostridium difficile
}

\begin{abstract}
Correspondence
Farah Babakhani

fbabakahni@optimerpharma.com
\end{abstract}

Received 22 December 2010

Accepted 18 February 2011

\author{
Farah Babakhani, Abraham Gomez, Nikki Robert and Pamela Sears \\ Optimer Pharmaceuticals, San Diego, CA, USA
}

\begin{abstract}
The kinetics of bacterial killing for fidaxomicin and its major metabolite, OP-1118, were investigated against Clostridium difficile strains, including two clinical strains belonging to the restriction endonuclease analysis group BI (ORG 1687 and 1698), the ATCC 43255 strain and two laboratory-derived mutant strains with decreased susceptibility to fidaxomicin (ORG 919 and 1620). Both fidaxomicin and OP-1118 demonstrated time-dependent killing of $C$. difficile strains. Fidaxomicin (at $4 \times$ MIC) reduced bacterial counts of the ATCC 43255 strain, clinical strain ORG 1687 and the two laboratory-generated mutant strains by $\geqslant 3$ logs within $48 \mathrm{~h}$ of exposure. The other BI strain, ORG 1698, was tested at $2 \times$ MIC fidaxomicin with bacterial counts decreasing $1 \log$ in 48 h. Exposure to OP-1118 (at $4 \times \mathrm{MIC}$ ) also resulted in a $\geqslant 3 \log$ drop in c.f.u. counts for the ATCC 43255 strain, the clinical BI strain ORG 1687 and the mutant strain ORG 919. Higher concentrations of OP-1118 ( $32 \times \mathrm{MIC})$ were required for a 3 log reduction in c.f.u. counts for the other BI strain, ORG 1698. In summary, the results indicate that both fidaxomicin and its major metabolite, OP-1118, are bactericidal against $C$. difficile strains, including the hypervirulent restriction endonuclease analysis group $\mathrm{BI}$ strains, at concentrations that are many fold below the detected faecal concentrations of these compounds after oral administration of fidaxomicin.
\end{abstract}

\section{INTRODUCTION}

Clostridium difficile is the leading cause of nosocomial infections in community hospitals, surpassing meticillinresistant Staphylococcus aureus infections in prevalence (Miller et al., 2010). Following several major outbreaks of C. difficile infection by hypervirulent strains in North America and Europe, the disease continues to be a major cause of diarrhoea in hospitalized patients and among the elderly in long-term care facilities. The mortality and morbidity associated with $C$. difficile infections is high, resulting in significant economic burden (Dubberke \& Wertheimer, 2009). Regardless of the impact, there has been little improvement in $C$. difficile treatment in the last 30 years. Current standard therapies are inadequate, the response rate to metronidazole has declined and there is a high rate of recurrence of infection following treatment with either metronidazole or vancomycin (Miller, 2010; Rupnik et al., 2009).

Fidaxomicin (formerly OPT-80 and PAR-101) is a novel, narrow-spectrum antibiotic in development for the treatment of C. difficile infection (Ackermann et al., 2004; Credito \& Appelbaum, 2004; Finegold et al., 2004). The putative target for fidaxomicin is the RNA polymerase (Talpaert et al., 1975). Although this target is shared by the rifamycin class of compounds, the site of action for fidaxomicin, which is structurally related to lipiarmycin, is thought to be distinct and there is no cross-resistance between rifampicin and fidaxomicin in C. difficile
(Babakhani et al., 2004). Furthermore, lipiarmycin is thought to act at the early stages of transcription and has been shown to inhibit the holoenzyme better than the core enzyme (Sonenshein \& Alexander, 1979).

Oral administration of both vancomycin and fidaxomicin results in a high faecal concentration of the drugs [ $>1 \mathrm{mg}$ ( faeces $)^{-1}$ ] with minimal systemic absorption (Johnson et al., 1992; Shue et al., 2008). Following oral administration (400 mg per day), fidaxomicin is excreted mainly in the faeces along with high concentrations of its major metabolite, OP-1118, which is derived by hydrolysis of the isobutyryl ester located at the $4^{\prime}$ position of fidaxomicin. Similar to the parental compound, OP-1118 demonstrates a narrow-spectrum activity against $C$. difficile, albeit with a 32-fold higher $\mathrm{MIC}_{90}$ (Babakhani et al., 2007). In recent phase 3 clinical trials in patients with C. difficile infection, fidaxomicin not only was well tolerated and achieved the primary end point of clinical cure but also demonstrated a significantly lower rate of recurrence of infection compared with vancomycin (Crook et al., 2010; Louie et al., 2009b; Miller et al., 2009). Analysis of stool samples from subjects during a phase 2 doseranging study demonstrated that fidaxomicin not only lacks activity against Gram-negative bacteria but is also sparing of Gram-positive commensals (Louie et al., 2009a; Tannock et al., 2010).

The narrow antimicrobial spectrum of activity of fidaxomicin has been well established by several studies; 
however, there are no data on the killing kinetics of the compound and its major metabolite (Ackermann et al., 2004; Credito \& Appelbaum, 2004; Finegold et al., 2004). This study investigated the killing rate of both fidaxomicin and OP-1118 in comparison with vancomycin in wild-type hypervirulent strains, as well as in laboratory-derived strains with reduced susceptibility to fidaxomicin.

\section{METHODS}

Bacterial strains. Clinical C. difficile strains ORG 1687 and 1698 belonging to the restriction endonuclease analysis group BI were collected from subjects in fidaxomicin phase 3 trials in Canada. C. difficile strains 9689 and 43255 were obtained from the ATCC. Laboratory-generated $C$. difficile strains with reduced susceptibility to fidaxomicin were derived either from wild-type ATCC 43255 strain through multiple passages in the presence of increasing concentrations of fidaxomicin (ORG 919) or from wild-type ATCC 9689 strain following a single exposure of the bacteria to high concentrations of fidaxomicin (ORG 1620). All strains were stored at $-80{ }^{\circ} \mathrm{C}$ and subcultured on $5 \%$ blood agar plates under anaerobic conditions ( $80 \%$ nitrogen, $10 \%$ carbon dioxide and $10 \%$ hydrogen) prior to testing.

Antimicrobial agents. Vancomycin was prepared as a $10 \mathrm{mg} \mathrm{ml} \mathrm{m}^{-1}$ stock in water. Similar stock concentrations of fidaxomicin and OP1118 were prepared by dissolving the compounds in DMSO. All drugs were further diluted to an appropriate concentration in growth medium (Brucella broth supplemented with vitamin $\mathrm{K}$ and haemin) before testing.

MIC determination and media. The Clinical and Laboratory Standards Institute microbroth (rather than agar) dilution method (recommended only for Bacteroides fragilis) was used with slight modifications for MIC determination because it more closely represents the conditions of the killing studies (CLSI, 2007). Lysed blood, which obscures the colour of the redox indicator resazurin in media, was omitted from the culture medium without affecting the growth of $C$. difficile. Briefly, microtitre plates containing tenfold serially diluted drugs were equilibrated inside an anaerobic glove box for a minimum of $3 \mathrm{~h}$. C. difficile $\left(10^{5}\right.$ c.f.u.) was added to each well and, after $48 \mathrm{~h}$ incubation at $35{ }^{\circ} \mathrm{C}$, the plates were examined for growth of $C$. difficile colonies. The MIC was determined as the drug concentration where no growth or the most significant reduction in growth was observed. MIC determinations were performed in duplicate. However, as MIC values can vary slightly between runs, duplicate MIC determination plates were also included in each killing kinetics experiment to confirm the MIC for that experiment.

Time-kill curve studies. All killing kinetics experiments were performed inside an anaerobic chamber. Colonies from blood agar plates were inoculated into Brucella broth supplemented with vitamin $\mathrm{K}$ and haemin and allowed to grow at $35^{\circ} \mathrm{C}$ until they reached a growth turbidity $\left(\mathrm{OD}_{600}\right)$ of 0.4 , which, based on our experience, corresponds to $\sim 1 \times 10^{8}$ cells ml ${ }^{-1}$. A portion of the cells $\left(\sim 1 \times 10^{6}\right.$ cells) was inoculated into $5 \mathrm{ml}$ supplemented Brucella broth containing fidaxomicin, OP-1118 or vancomycin at concentrations that were multiples of the MIC. A no-drug (control) tube was also included in each run. At different time intervals over a $48 \mathrm{~h}$ incubation period, aliquots from each treatment were removed and serially (tenfold) diluted, and $100 \mu \mathrm{l}$ of the dilution was plated onto duplicate blood agar plates. The number of c.f.u. was determined after incubation of the plates at $35{ }^{\circ} \mathrm{C}$ for $48 \mathrm{~h}$. With this method, the threshold of detection was 100 c.f.u. $\mathrm{ml}^{-1}$, or a single colony when $100 \mu \mathrm{l}$ of a tenfold-diluted culture was plated. Killing kinetics experiments for the most part were performed once for each strain and drug combination (at multiples of the MIC) except as specified in Results (in duplicate for OP-1118 against ORG 1698 or triplicate for fidaxomicin against ORG 919).

The problem of residual drug carryover was addressed by dilution of cultures at least tenfold prior to plating, i.e. once plated, the drug would diffuse and be diluted a further 250 -fold, based on a $100 \mu \mathrm{l}$ inoculum and a $25 \mathrm{ml}$ plate volume. To confirm the lack of effect of residual drug on growth, a broth culture of a $C$. difficile ATCC strain was grown overnight and $50 \mu \mathrm{l}$ was removed at different time points, serially diluted and mixed with an equal volume of the drug (final concentration $4-8 \times$ MIC) immediately prior to plating. C.f.u. counts with and without exposure to the drug were compared to verify that dilution steps during plating led to negligible drug concentrations that did not affect the growth of the bacteria. Assessment of the impact of drug carryover was performed once with both the ATCC 43255 and 9689 strains.

\section{RESULTS AND DISCUSSION}

\section{MIC values}

The MIC values for each strain, determined by the broth microdilution method, are summarized in Table 1. Both clinical strains had fidaxomicin MICs of $0.25 \mathrm{mg} \mathrm{l}^{-1}$ and OP-1118 MIC values of 2-4 mg $\mathrm{l}^{-1}$. The ATCC strains 43255 and 9689 demonstrated fidaxomicin MICs of 0.016 0.25 and $0.016 \mathrm{mg} \mathrm{l}^{-1}$, respectively, and OP-1118 MICs of 4 and $0.25 \mathrm{mg} \mathrm{l}^{-1}$, respectively. The laboratory strains generated from the ATCC strains demonstrated reduced susceptibility to both fidaxomicin $\left(2-4 \mathrm{mg} \mathrm{l}^{-1}\right.$ for ORG 919 and $1 \mathrm{mg} \mathrm{l}^{-1}$ for ORG 1620) and OP-1118 (128 mg $1^{-1}$ for ORG 919). The vancomycin MIC for all strains, with the exception of ORG 919, was $1 \mathrm{mg} \mathrm{l}^{-1}$. The MIC for ORG 919 was fourfold higher than that for the parental ATCC strain 43255 at $4 \mathrm{mg} \mathrm{l}^{-1}$.

\section{Killing kinetics}

The killing kinetics of fidaxomicin and OP-1118 for each strain are described below and shown separately in Figs 1-5.

To demonstrate lack of effect of drug carryover on growth, bacterial counts from growing broth cultures of $C$. difficile

Table 1. MIC values $\left(\mathrm{mg} \mathrm{l}^{-1}\right)$ for fidaxomicin, OP-1118 and vancomycin

ND, Not done.

\begin{tabular}{|c|c|c|c|c|c|c|}
\hline \multirow[t]{3}{*}{ Antibiotic } & \multicolumn{2}{|c|}{ ATCC strains } & \multicolumn{2}{|c|}{$\begin{array}{l}\text { Clinical } \\
\text { strains }\end{array}$} & \multicolumn{2}{|c|}{$\begin{array}{l}\text { Laboratory } \\
\text { strains }\end{array}$} \\
\hline & 43255 & 9689 & ORG & ORG & ORG & ORG \\
\hline & & & 1687 & 1698 & 919 & 1620 \\
\hline Fidaxomicin & $0.25,0.06$ & 0.016 & 0.25 & 0.25 & 2,4 & 1 \\
\hline OP-1118 & 4 & 0.25 & 2 & 2,4 & 128 & ND \\
\hline Vancomycin & 1 & 1 & 1,2 & 1 & 4 & 1 \\
\hline
\end{tabular}




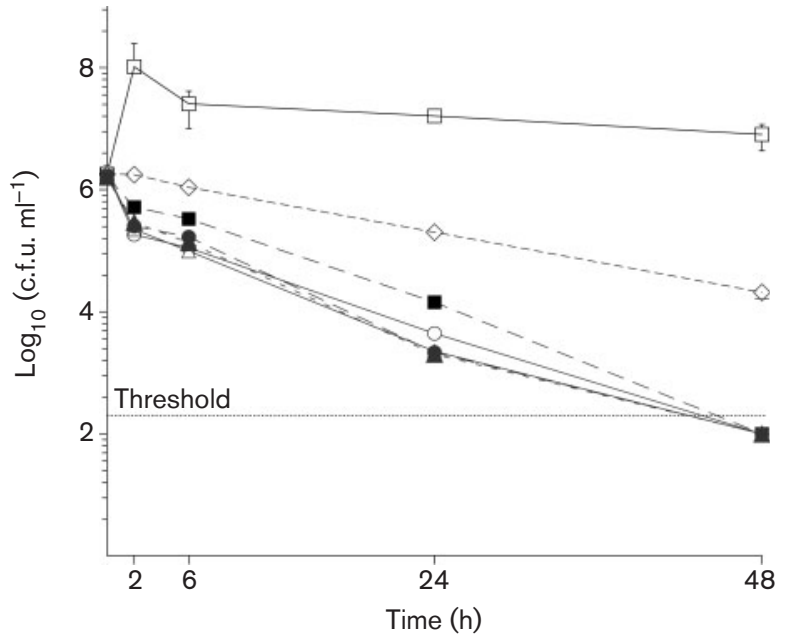

Fig. 1. Killing kinetics of fidaxomicin ( $\bigcirc, 4 \times \mathrm{MIC} ; \triangle, 32 \times \mathrm{MIC})$, OP-1118 ( $\mathbf{\Lambda}, 2 \times \mathrm{MIC} ; \bullet, 4 \times \mathrm{MIC} ; \mathbf{\square}, 16 \times \mathrm{MIC})$ and vancomycin $(\diamond, 4 \times$ MIC $)$ against $C$. difficile ATCC 43255. A no-drug control $(\square)$ was included with each run. Samples of $<100$ c.f.u. $\mathrm{ml}^{-1}$ were below the limit of detection.

ATCC 43255 or 9689 were determined at various time points by serial dilution followed by mixing with equal volume of $8 \times$ or $16 \times$ concentrations or no drug immediately prior to plating. Exposure of cells to $4-8 \times$ MIC concentrations of drugs during serial dilution and plating did not affect the viability of cells. The numbers of c.f.u. were similar at each time point whether the cells were mixed with high concentrations of the drugs or not.

The killing kinetics against C. difficile ATCC 43255 are depicted in Fig. 1. Both fidaxomicin and its major metabolite, OP-1118, at $4 \times$ MIC exerted bactericidal activity against this strain; bacterial counts dropped below the detection limit $\left(100\right.$ c.f.u. $\left.\mathrm{ml}^{-1}\right)$ by $48 \mathrm{~h}$ for both

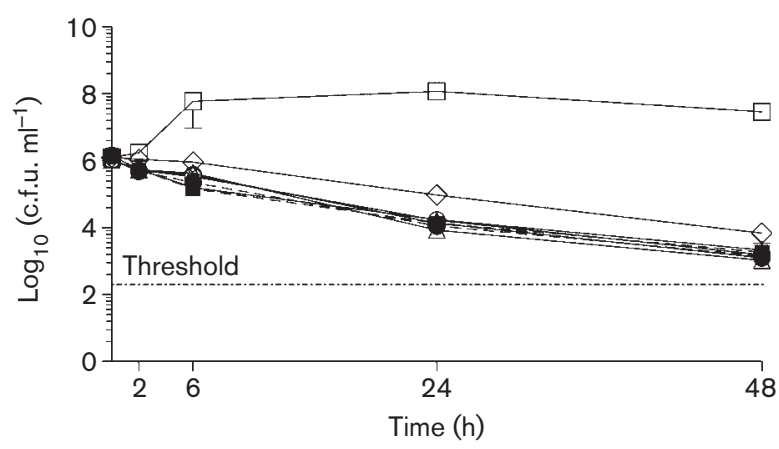

Fig. 2. Killing kinetics of fidaxomicin $(\triangle, 2 \times \mathrm{MIC} ; \bigcirc, 4 \times \mathrm{MIC}$; $\otimes, 8 \times \mathrm{MIC}), \mathrm{OP}-1118(\boldsymbol{\Delta}, 4 \times \mathrm{MIC} ; \bullet, 8 \times \mathrm{MIC} ; \mathbf{\square}, 16 \times \mathrm{MIC})$ and vancomycin $(\diamond, 2-4 \times \mathrm{MIC})$ against $C$. difficile clinical $\mathrm{BI}$ strain ORG 1687. A no-drug control $(\square)$ was included with each run. Samples of $<100$ c.f.u. $\mathrm{ml}^{-1}$ were below the limit of detection.

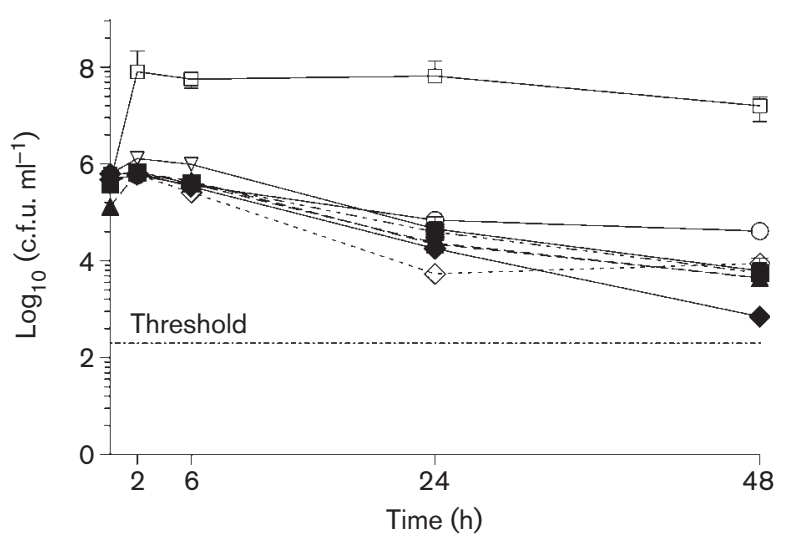

Fig. 3. Killing kinetics of fidaxomicin ( $\bigcirc, 2 \times \mathrm{MIC})$, OP-1118 ( $\boldsymbol{\Delta}, 4 \times \mathrm{MIC} ; 0,8 \times \mathrm{MIC} ; \mathbf{\square}, 16 \times \mathrm{MIC} ;, 32 \times \mathrm{MIC})$ and vancomycin $(\diamond, 2 \times \mathrm{MIC})$ against $C$. difficile clinical BI strain ORG 1698. A no-drug control $(\square)$ was included with each run. Samples of $<100$ c.f.u. $\mathrm{ml}^{-1}$ were below the limit of detection.

compounds. OP-1118 was also bactericidal at $2 \times$ MIC. In contrast, cells exposed to vancomycin at $4 \times$ MIC were, as expected, killed more slowly and the bacterial count dropped by only 2 logs within $48 \mathrm{~h}$.

Similarly, both fidaxomicin (even at $2 \times$ MIC) and OP1118 demonstrated time-dependent bactericidal activity against the clinical BI strain ORG 1687 (Fig. 2). The number of c.f.u. dropped $3 \operatorname{logs}$ by $48 \mathrm{~h}$ in the presence of fidaxomicin or OP-1118. In contrast, killing activity by vancomycin at $4 \mu \mathrm{g} \mathrm{ml}^{-1}$ was slower, dropping only $1 \mathrm{log}$ in $24 \mathrm{~h}$ and slightly over 2 logs in $48 \mathrm{~h}$.

The killing activity of fidaxomicin against the other clinical BI strain, ORG 1698, was tested once at $2 \times$ MIC, with bacterial counts dropping slightly more than $1 \log$ by $48 \mathrm{~h}$

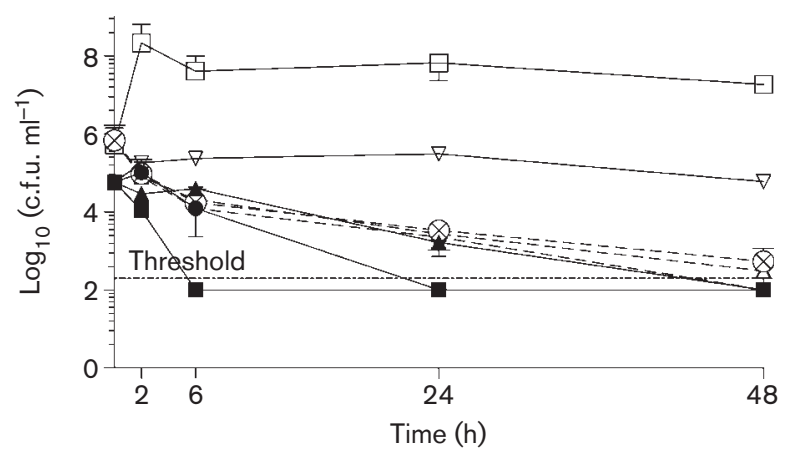

Fig. 4. Killing kinetics of fidaxomicin $(\bigcirc, 4-8 \times \mathrm{MIC} ; \triangle, 16 \times \mathrm{MIC}$; $\otimes, 32 \times \mathrm{MIC}), \mathrm{OP}-1118$ ( $\boldsymbol{\Delta}, 4 \times \mathrm{MIC} ; \bullet, 8 \times \mathrm{MIC} ; \boldsymbol{\square}, 16 \times \mathrm{MIC})$ and vancomycin $(\nabla, 4 \times \mathrm{MIC})$ against laboratory-generated mutant C. difficile 919 with reduced susceptibility to fidaxomicin. A nodrug control $(\square)$ was included with each run. Samples of $<100$ c.f.u. $\mathrm{ml}^{-1}$ were below the limit of detection. 


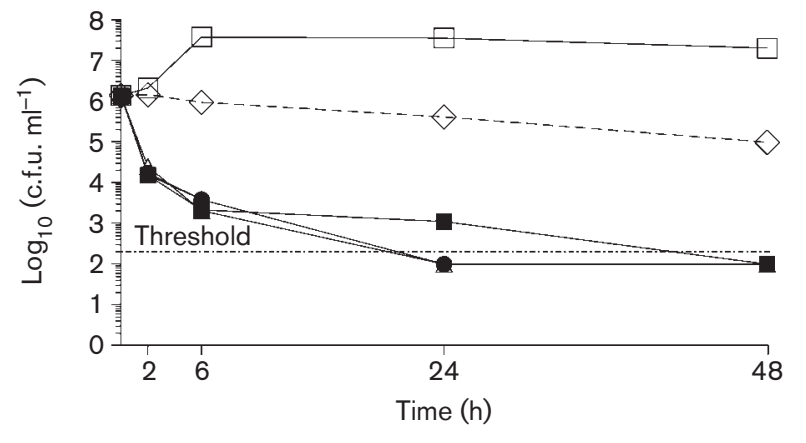

Fig. 5. Killing kinetics of fidaxomicin $(\bigcirc, 2 \times \mathrm{MIC} ; \triangle, 4 \times \mathrm{MIC}$; $\bullet, 8 \times \mathrm{MIC} ; \boldsymbol{\square}, 16 \times \mathrm{MIC})$ and vancomycin $(\diamond, 4 \times \mathrm{MIC})$ against laboratory-generated mutant $C$. difficile 1620 with reduced susceptibility to fidaxomicin. A no-drug control $(\square)$ was included with each run. Samples of $<100$ c.f.u. $\mathrm{ml}^{-1}$ were below the limit of detection.

(Fig. 3). In the same experiment, however, c.f.u. counts for $2 \times$ MIC vancomycin-treated cells initially dropped by $\sim 2$ logs within $24 \mathrm{~h}$ and then began to recover, and by $48 \mathrm{~h}$ the c.f.u. count was $<2$ logs lower than the initial inoculum, indicating a lack of efficient killing. OP-1118 bactericidal activity against $C$. difficile ORG 1698 could not be determined reliably; inocula in duplicate experiments were insufficient such that the final counts were below the limit of detection. Nevertheless, bacterial counts dropped by at least 2 logs at 4-16 $\times$ MIC and by more than 2 logs at $32 \times$ MIC.

Similar to the wild-type parental strain ATCC 43255, the mutant strain ORG 919 was killed by fidaxomicin and OP1118, with c.f.u. counts dropping below the detection threshold by $48 \mathrm{~h}$ (Fig. 4). A 3 log drop in c.f.u. counts could not be determined reliably for this organism, as inoculum densities were lower than anticipated during the three separate trials. Vancomycin at $4 \times$ MIC inhibited c.f.u. counts by $1 \log$ at $48 \mathrm{~h}$.

Fidaxomicin demonstrated a $>3 \log$ drop in c.f.u. at concentrations as low as $2 \times \mathrm{MIC}$ against the mutant strain ORG 1620 (Fig. 5). In contrast, vancomycin killing at $4 \times$ MIC was slow and the c.f.u. counts dropped only slightly more than $1 \log$ in $48 \mathrm{~h}$. The bactericidal activity of OP1118 against this mutant strain was not investigated.

The results of this investigation demonstrated that both fidaxomicin and its metabolite OP-1118 have bactericidal activity against $C$. difficile strains in vitro. Both ATCC and clinical strains were killed by fidaxomicin, with c.f.u. counts dropping significantly for ATCC strain 43255 (>3 log drop) and the clinical BI strain ORG 1687 (3 log drop). The bactericidal activity of fidaxomicin against the other clinical BI strain, ORG 1698, could not be established in this report as it was not tested above $2 \times \mathrm{MIC}$ concentrations; at $1 \times$ and $2 \times$ MIC concentrations, however, the bacterial count for this strain dropped by $1 \mathrm{log}$ in $24 \mathrm{~h}$ and declined only slightly thereafter.
This report further demonstrates that fidaxomicin's major metabolite, OP-1118, is also bactericidal in a timedependent manner. Both ATCC strain 43255 and the clinical BI strain ORG 1687 were killed in the presence of a $4 \times$ MIC concentration of OP-1118. Similar to the parental compound, the bactericidal activity against the ATCC strain was more pronounced $(>3 \operatorname{logs})$ than against clinical BI strains ORG 1687 (3 logs) and ORG 1698 (2 logs). For the latter strain, a higher concentration of metabolite $(32 \times \mathrm{MIC})$ was required to obtain a $3 \mathrm{log}$ reduction in c.f.u. counts.

Bactericidal activity of fidaxomicin was also observed against the laboratory-generated mutant strains with increased fidaxomicin MIC values. ORG 919 (MIC 2$4 \mu \mathrm{g} \mathrm{ml}^{-1}$ ) was derived in our laboratory from C. difficile ATCC 43255 after multiple passages in the presence of increasing concentrations of fidaxomicin and had two mutations in the $r p o C$ region of the RNA polymerase $\left(\mathrm{Gln}^{781} \rightarrow\right.$ Arg and Asp $\left.{ }^{1127} \rightarrow \mathrm{Glu}\right)$ (J. Seddon, Optimer, personal communication). ORG 1620 with increased fidaxomicin MIC (1 mg $\left.\mathrm{l}^{-1}\right)$ was generated through a single-step exposure of ATCC 9689 to a high concentration of fidaxomicin and had a single mutation in the $r p o B$ region of the RNA polymerase $\left(\mathrm{G} / n^{1074} \rightarrow\right.$ Lys). The latter amino acid mutation has been identified at homologous positions in other species with a low susceptibility to lipiarmycin, a related macrocyclic compound (Kurabachew et al., 2008; Mekler et al., 2004). Despite a several-fold higher MIC compared with the parental strains, both strains with mutations in the RNA polymerase were rapidly killed by fidaxomicin at $4 \times$ MIC concentrations, indicating that mutants that may arise during fidaxomicin therapy will probably be eliminated by the high levels of drug achieved in the gut.

In summary, the results of this study confirmed that fidaxomicin and its major metabolite, OP-1118, are bactericidal against $C$. difficile, including hypervirulent strains, whereas the other leading drug used, vancomycin, is bacteriostatic against C. difficile (Odenholt et al., 2007). It is expected that with high concentrations of both compounds, reproducibly obtained in the gut, even mutant strains with increased fidaxomicin MIC values are likely to be killed effectively during therapy.

\section{ACKNOWLEDGEMENTS}

This work was supported by a Public Health grant from the National Institutes of Health.

\section{REFERENCES}

Ackermann, G., Löffler, B., Adler, D. \& Rodloff, A. C. (2004). In vitro activity of OPT-80 against Clostridium difficile. Antimicrob Agents Chemother 48, 2280-2282.

Babakhani, F. K., Shangle, S., Robert, N., Sears, P. \& Shue, Y. K. (2004). Resistance development, cross-resistance, and synergy 
studies of OPT-80. In Abstracts of the 44th Interscience Conference on Antimicrobial Agents and Chemotherapy, Washington, DC, USA, abstract E-2047. Washington, DC: American Society for Microbiology.

Babakhani, F., Seddon, J., Robert, N., Gomez, A. \& Sears, P. (2007). Narrow spectrum activity and low fecal binding of OPT-80 and its major hydrolysis metabolite (OP-1118). In Abstracts of the 47th Interscience Conference on Antimicrobial Agents and Chemotherapy, Chicago, IL, USA, abstract E-2076. Washington, DC: American Society for Microbiology.

CLSI (2007). Methods for Antimicrobial Susceptibility Testing of Anaerobic Bacteria, 7th edn; Approved Standard. M11-A7. Wayne, PA: Clinical and Laboratory Standards Institute.

Credito, K. L. \& Appelbaum, P. C. (2004). Activity of OPT-80, a novel macrocycle, compared with those of eight other agents against selected anaerobic species. Antimicrob Agents Chemother 48, 44304434.

Crook, D., Peto, T., Miller, M., Louie, T., Cornely, O., Shue, Y. K. \& Gorbach, S. (2010). Efficacy and safety of fidaxomicin (FDX) vs vancomycin (VAN) in Clostridium difficile infection (CDI) in 2 randomized controlled trials (RCT) with 1105 patients. In Abstracts of the 48th Infectious Disease Society of America Annual Meeting, Vancouver, Canada, abstract 1417. Arlington, VA: Infectious Disease Society of America.

Dubberke, E. R. \& Wertheimer, A. I. (2009). Review of current literature on the economic burden of Clostridium difficile infection. Infect Control Hosp Epidemiol 30, 57-66.

Finegold, S. M., Molitoris, D., Vaisanen, M. L., Song, Y., Liu, C. \& Bolaños, M. (2004). In vitro activities of OPT-80 and comparator drugs against intestinal bacteria. Antimicrob Agents Chemother 48, 4898-4902.

Johnson, S., Homann, S. R., Bettin, K. M., Quick, J. N., Clabots, C. R., Peterson, L. R. \& Gerding, D. N. (1992). Treatment of asymptomatic Clostridium difficile carriers (fecal excretors) with vancomycin or metronidazole. A randomized, placebo-controlled trial. Ann Intern Med 117, 297-302.

Kurabachew, M., Lu, S. H., Krastel, P., Schmitt, E. K., Suresh, B. L., Goh, A., Knox, J. E., Ma, N. L., Jiricek, J. \& other authors (2008). Lipiarmycin targets RNA polymerase and has good activity against multidrug-resistant strains of Mycobacterium tuberculosis. J Antimicrob Chemother 62, 713-719.

Louie, T. J., Emery, J., Krulicki, W., Byrne, B. \& Mah, M. (2009a). OPT-80 eliminates Clostridium difficile and is sparing of Bacteroides species during treatment of $C$. difficile infection. Antimicrob Agents Chemother 53, 261-263.
Louie, T. J., Mullane, K. M., Weiss, K., Lentnek, A., Golan, Y., Gorbach, S., Sears, P., Shue, Y. K. \& Miller, M. A. (2009b). A randomised, doubleblind clinical trial of OPT-80 versus vancomycin in Clostridium difficile infection. In Abstracts of the 19th European Congress of Clinical Microbiology and Infectious Diseases, Helsinki, Finland, abstract 0148. Basel, Switzerland: European Society of Clinical Microbiology and Infectious Diseases.

Mekler, V., Sineva, E., Mukhopadhyay, J., Sajida, I., Wang, X., Kortkhonja, E., Donadio, S. \& Ebright, R. (2004). Target and mechanism of the transcription inhibitor lipiarmycin. Presented at the Biophysical Society 48th Annual Meeting, Baltimore, MD, USA.

Miller, M. (2010). Fidaxomicin (OPT-80) for the treatment of Clostridium difficile infection. Expert Opin Pharmacother 11, 15691578.

Miller, M. A., Mullane, K. M., Weiss, K., Lentnek, A., Golan, Y., Gorbach, S., Sears, P., Shue, Y. K. \& Louie, T. J. (2009). OPT-80 versus vancomycin in Clostridium difficile infection: results of a randomized clinical trial. Gastroenterology 136 (Suppl. 1), A-115.

Miller, B. A., Chen, L. F., Sexton, D. J. \& Anderson, D. J. (2010). The impact of hospital-onset healthcare facility associated Clostridium difficile infection (CDI) in community hospitals: surpassing methicillin-resistant Staphylococcus aureus (MRSA) as the new superbug. In Program and Abstracts of the 5th Decennial International Conference on Healthcare-Associated Infections (ICHAI), Atlanta, Georgia, abstract 386.

Odenholt, I., Walder, M. \& Wullt, M. (2007). Pharmacodynamic studies of vancomycin, metronidazole and fusidic acid against Clostridium difficile. Chemotherapy 53, 267-274.

Rupnik, M., Wilcox, M. H. \& Gerding, D. N. (2009). Clostridium difficile infection: new developments in epidemiology and pathogenesis. Nat Rev Microbiol 7, 526-536.

Shue, Y. K., Sears, P. S., Shangle, S., Walsh, R. B., Lee, C., Gorbach, S. L., Okumu, F. \& Preston, R. A. (2008). Safety, tolerance, and pharmacokinetic studies of OPT- 80 in healthy volunteers following single and multiple oral doses. Antimicrob Agents Chemother 52, 1391-1395.

Sonenshein, A. L. \& Alexander, H. B. (1979). Initiation of transcription in vitro inhibited by lipiarmycin. J Mol Biol 127, 55-72.

Talpaert, M., Campagnari, F. \& Clerici, L. (1975). Lipiarmycin: an antibiotic inhibiting nucleic acid polymerases. Biochem Biophys Res Commun 63, 328-334.

Tannock, G. W., Munro, K., Taylor, C., Lawley, B., Young, W., Byrne, B., Emery, J. \& Louie, T. (2010). A new macrocyclic antibiotic, fidaxomicin (OPT-80), causes less alteration to the bowel microbiota of Clostridium difficile-infected patients than does vancomycin. Microbiology 156, 3354-3359. 